Relations industrielles

Industrial Relations

\title{
Managerial Long-Range Planning, edited by George A. Steiner, McGraw-Hill Co., New York, 1963, 334 pages.
}

\section{Laurent Bélanger}

Volume 20, numéro 2, 1965

URI : https://id.erudit.org/iderudit/027572ar

DOI : https://doi.org/10.7202/027572ar

Aller au sommaire du numéro

Éditeur(s)

Département des relations industrielles de l'Université Laval

ISSN

0034-379X (imprimé)

1703-8138 (numérique)

Découvrir la revue

Citer ce compte rendu

Bélanger, L. (1965). Compte rendu de [Managerial Long-Range Planning, edited by George A. Steiner, McGraw-Hill Co., New York, 1963, 334 pages.] Relations industrielles / Industrial Relations, 20(2), 396-397.

https://doi.org/10.7202/027572ar

Tous droits réservés @ Département des relations industrielles de l'Université Laval, 1965
Ce document est protégé par la loi sur le droit d'auteur. L'utilisation des services d'Érudit (y compris la reproduction) est assujettie à sa politique d'utilisation que vous pouvez consulter en ligne.

https://apropos.erudit.org/fr/usagers/politique-dutilisation/ 


\section{RECENSION S BOOKS REVIEW}

Automation and Collective Bargaining, por Benjamin S. Kirsh, New York, Central Book Company, 1964, 219 pages.

L'auteur se pose d'abord quelques questions qui pourraient se résumer surtout à celle-ci: est-ce que la négociation collective elle-même survivra à tous ces changements technologiques, comme cadre principal de la règlementation des conditions de travail? Malgré tous les éléments de solutions que la négociation peut nous présenter aujourd'hui, on peut se demander si ce ne sont pas seulement des résultats valables en courte période, qui rendent difficile l'adaptation globale à des problèmes plus sérieux en longue période. Considérant les avantages et désavantages de l'automation tant du point de vue économique que social, Kirsh nous présente la négociation collective actuelle comme le moyen pratique de faire le moins de dommage possible économiquement et socialement.

Tout en montrant comment les lois ont contribué à rendre possible l'équilibre ou niveau du pouvoir de négociation entre les parties, il fait voir que le contexte d'outomation dans lequel nous vivons évolue rapidement, ce qui rend possible un débalancement dans le pouvoir de négociation.

Dans le passé, on peut voir l'évolution du syndicalisme cherchant à contrôler l'offre d'emploi, à l'aide de décisions juridiques découlant de certaines causes où les syndicats étaient accusés de violation de lois, par exemple des lois anti-trusts. L'auteur soulève le problème à savoir si la position monopolistique du travail organisé ne s'affaiblit pos ovec l'introduction de l'automation, après avoir été renforcée par certaines décisions en justice favorisant les syndicats.

Après une énumération de raisons acceptobles pour expliquer lo présence de plus en plus grande du gouvernement à la table de négociation, il y a une série d'exemples servant à démontrer que dans les secteurs les plus automatisés le pouvoir effectif de faire la grève semble amoindri. Avec l'apparition de nouveaux problèmes posés par l'automation, il $y$ a aussi une évolution dans les concepts de la direction des entreprises, ainsi les prérogatives de la gérance doivent changer en face des intérêts nouveaux de l'autre partie à la négociation.

Cet ouvroge étudie brièvement la portée de certaines clauses de la convention collective dans un contexte d'automation. On y met l'accent sur les clauses d'ancienneté surtout.

Certains éléments nouveaux viennent enrichir les systèmes de détermination de la rémunération par exemple le facteur d'amélioration annuelle du travailleur.

Selon Kirsh, le problème de déterminer la grandeur de l'unité de négociation la plus appropriée sera très important dans l'avenir.

L'attitude des syndicats de métier et celle des syndicats industriels en face des changements technologiques n'est pas la même. Le chapitre traitant de ces attitudes souligne l'importance des règles praticues de travail, que les travailleurs eux-mêmes s'imposent en face de certaines situations.

Cet ouvrage ne règle pas les problèmes nouveaux qui se présentent dans la négociation collective comme conséquence de l'automation. Mais on y retrouve une certaine objectivité en face des attitudes qui déterminent souvent le climat des négociations.

\section{Gilbert L'Heureux}

Managerial Long-Range Planning, edited by George A. Steiner, McGraw-Hill Co., New York, 1963. 334 pages.

Ce volume est le fruit d'un séminaire tenu à l'Université de California où des hommes d'affaires et des universitaires ont présenté leurs opinions sur les principes et pratiques de la planification en longue période au sein de l'entreprise.

Des représentants de quatorze grandes entreprises privées et publiques ont décrit la démarche utilisée dans le processus de planification.

Le deuxième chapitre intitulé: «Highlights of the Seminar » présente un résumé des principaux points qui ressortent de la discussion: 
1-La définition de la planification en longue période.

2-Les principes majeurs concernant l'organisation qu'il faut mettre sur pied en vue $d^{\prime} u n e$ planification fructueuse.

3-La démarche à suivre dans l'édification d'un plan.

4-La relation entre les objectifs de l'entreprise et le plan.

5--La détermination de la période couverte par le plan.

6-L'utilisation des techniques quantitatives en vue d'améliorer le processus et les résultats.

7-Les problèmes majeurs auxquels fait face actuellement la planification.

Ceux qui prirent part à la discussion tombèrent d'accord sur la nécessité de la planification et de la coordination des plans de courte période avec ceux de longue-période. La flexibilité et la créativité sont deux conditions essentielles dans l'élaboration d'un plan. L'expérience démontre que des plans couvrant une période de cinq ans sont les plus pratiques.

Co volume apporte un complément valable oux principes et aux expériences pratiques qui sont présentées dans les manuels en administration des entreprises.

\section{Laurent BELANGER}

Economics of Labor Relations. By Gordon F. Bloom and Herbert R. Northrup. Fifth edition. Richard D. Irwin, Inc., Homewood, Illinois, 1965, 911 pp.

Cet ouvrage connait une cinquième édition cans l'espace de quinze ans. C'est déjà un signe manifestant la valeur d'un tel monuel.

Les auteurs l'ont mis à jour en tenant compte des développements dans les relations du travail de même que des nouveaux problèmes qui ont surgi depuis quelques années: The Civil Rights Act de 1964, le chômage, la reconversion industrielle, les nouvelles formules de négociation collective; le plan Kaiser, etc.

Le dernier chapitre est consacré aux problèmes du travail dans les années soixante: sécurité sociale, nécessité de nouveaux concepts, intervention gouvernementale, nécessité de redéfinir le rôle des syndicats et de la direction des entreprises comme celui de l'industrie et du gouvernement.
Ce chapitre se borne seulement à poser les problèmes dans des termes assez ternes et n'apporte rien de bien substantiel. Les auteurs ne semblent point pouvoir se dégager de conceptions dépassées qu'ils reprochent, avec raison, au syndicalisme ouvrier.

Entre les mains de professeurs dynamiques et informés, ce manuel peut être un bon instrument. A la fin de chaque chapitre on propose des questions pour discussions et on présente une bibliographie sélective utile à l'étudiant pour compléter ses études.

\section{Gérard DION}

Readings on Economic Sociology, Edited by Neil J. Smelzer, Readings in Modern Sociology Series, Prentice-Hall, Inc., Englewood Cliffs, New Jersey, 1965, 303 pages.

\section{The Social Context of Economic Behavior,} par W.T. Tucker Holt, Rinehart and Winston, Inc., 1964, 163 pages.

Bien que la sociologie et l'économique possèdent une certaine autonomie sur le plan des concepts, des hypothèses et de la démarche intellectuelle, on ne peut pour autant nier l'existence des points de rencontre entre ces deux disciplines.

Ces deux auteurs se sont assignés la tâche de faire ressortir ces points en montrant la façon dont le comportement économique s'insère dans un contexte social beaucoup plus vaste où des facteurs culturels, psychologiques, organisationnels, politiques, viennent s'ajouter aux variables économiques pour arriver à une meilleure explication de la réalité sociale.

\section{Variables culturelles et activité économique}

Pour cette première section, Smelzer a fait un choix de lecteurs qui mettent à jour les relations entre la structure économique et la vie culturelle de la société. En effet, un premier article, celui de Birnbaum, examine de nouveau les théories de Marx et de Weber sur I'origine du capitalisme. Dans un autre article, Bendix retrace brièvement les changements dans le contenu des idéologies anglo-américaines et russes, la signification historique des idéologies directoriales, les différences entre les formes totalitaires et non-totalitaires de la subordination dans la société industrielle.

Le traitement des variables culturelles chez Tucker se situe à un niveau beaucoup plus concret. L'activité économique influence la culture d'une société et vice-versa. Par 